\title{
TIKHONOV REGULARIZATION WITH OVERSMOOTHING PENALTY FOR NON-LINEAR ILL-POSED PROBLEMS IN HILBERT SCALES
}

\author{
BERND HOFMANN AND PETER MATHÉ
}

\begin{abstract}
We study Tikhonov regularization for ill-posed nonlinear operator equations in Hilbert scales. Our focus is on the interplay between the smoothness-promoting properties of the penalty and the smoothness inherent in the solution. The objective is to study the situation when the unknown solution fails to have a finite penalty value, hence when the penalty is oversmoothing. By now this case was only studied for linear operator equations in Hilbert scales. We extend those results to certain classes of nonlinear problems. The main result asserts that, under appropriate assumptions, order optimal reconstruction is still possible. In an appendix we highlight that the non-linearity assumption underlying the present analysis is met for specific applications.
\end{abstract}

\section{INTRODUCTION}

Tikhonov regularization is a versatile means of stabilizing linear and non-linear ill-posed operator equations in Hilbert and Banach spaces. In either case a stable approximate solution is obtained by minimizing the Tikhonov functional, which consists of two summands: a term representing the data misfit and a stabilizing penalty. There is vast literature on the quality of the obtained approximate solutions, and we mention the monographs $[8,25,26,28]$. The analysis always uses the minimizing property, i.e., that the Tikhonov functional at the minimizer takes smaller values than at the true solution.

The choice of the penalty is context-dependent. This has consequences for the obtained approximations, because the corresponding mimimizers will have a finite penalty value. Some of the relevant choices are total-variation penalties, often used in image reconstruction (cf., e.g., [5]), since such penalties promote edges, and $\ell^{1}$-penalties (cf., e.g., [7]) and $[4,10,18]$ ) or $L^{1}$-penalties (cf., e.g., $[3,6]$ and $[25$, p. 87-89]), since they have sparsity promoting properties.

Actually, a smoothing penalty may be used in order to detect whether a solution has certain smoothness properties. This was studied in the context of numerical differentiation in [30] (see also [29] for extensions). These authors show that, for a prescribed choice of the regularization parameter, the penalty must blow up whenever the solution fails to have a finite penalty value, and this can be used to detect singularities. 
Within the present study the objective is to handle penalties which impose smooth reconstructions. One reason to do so is the well-known fact that the classical version of Tikhonov regularization has limited qualification, which means that it cannot take into account higher solution smoothness. Hence its error norm convergence rate as a function of the noise level $\delta$ cannot exceed $\delta^{2 / 3}$ or $\delta^{1 / 2}$, depending on whether a priori or a posteriori parameter choice is used.

In all the above cases we face the problem that the unknown solution may not have the expected feature implied by the penalty. Images may not have only a few sharp edges, solutions may not have a sparse representation or may not be smooth. Then the question arises whether the use of such mis-specified penalties still allows for good reconstructions yielding errors of optimal order with respect to the noise level. Here we focus on smoothness-promoting penalties, and we shall call the penalty oversmoothing if the unknown solution element fails to have the expected smoothness, and hence the penalty will not have a finite value at the solution.

For linear ill-posed problems in Hilbert scales F. Natterer has shown in the seminal paper [21] that oversmoothing makes no trouble and corresponding results on convergence rates have been established. The analysis of linear ill-posed problems in Hilbert scales is special, because the minimizer of the Tikhonov functional is given explicitly and the analysis is based on the explicit representation.

Here we extend those results to some classes of non-linear operator equations, which have only solutions with infinite penalty value, and hence we cannot apply the arguments, typically used in the proofs. Our 'proof architecture' is also different from that of [21], even in the special case of a linear forward operator, because it uses the discrepancy principle for the choice of the regularization parameter. The present approach may be regarded as a further step towards regularization theory for linear or non-linear operator equations with oversmoothing penalty.

Specifically, we consider the approximate solution of an (at least locally) ill-posed operator equation

$$
F(x)=y
$$

with an (in general) non-linear forward operator $F: \mathcal{D}(F) \subseteq X \rightarrow Y$ between the infinite dimensional Hilbert spaces $X$ and $Y$, with domain of definition $\mathcal{D}(F)$. By $x^{\dagger}$ we denote a solution to (1) for given $y$.

In order to consider a Hilbert scale, and to treat an oversmoothing penalty, we introduce an unbounded linear self-adjoint operator $B: \mathcal{D}(B) \subset X \rightarrow X$, which is strictly positive such that we have for some $m>0$

$$
\|B x\|_{X} \geq m\|x\|_{X}, \quad \text { for all } \quad x \in \mathcal{D}(B) .
$$


Based on noisy data $y^{\delta} \in Y$, obeying the deterministic noise model

$$
\left\|y-y^{\delta}\right\|_{Y} \leq \delta
$$

with noise level $\delta>0$, we use regularized solutions $x_{\alpha}^{\delta} \in \mathcal{D}$ for

$$
\mathcal{D}:=\mathcal{D}(F) \cap \mathcal{D}(B),
$$

being minimizers of the extremal problem

(4) $T_{\alpha}^{\delta}(x):=\left\|F(x)-y^{\delta}\right\|_{Y}^{2}+\alpha\|B(x-\bar{x})\|_{X}^{2} \rightarrow \min$, subject to $x \in \mathcal{D}$,

for the Tikhonov functional $T_{\alpha}^{\delta}$, where $\bar{x} \in \mathcal{D}$ is a given smooth reference element.

We shall focus on the oversmoothing case, i.e., when $x^{\dagger} \notin \mathcal{D}(B)$ such that $T_{\alpha}^{\delta}\left(x^{\dagger}\right)=\infty$.

As already mentioned, in the linear case, i.e. for a bounded linear operator $F$, such approach was studied by Natterer in [21]. In a slightly different setup oversmoothing was studied in [20]. Again, optimality was shown for a priori parameter choice and for several a posteriori choices of the regularization parameter $\alpha$.

The outline is as follows. We formulate our assumptions and the main result in Section 2. The proof of this result is then given in Section 3. Relevant outcomes of this study are discussed in Section 4. The Appendix provides us with several assertions and examples in order to validate the required non-linearity assumption in specific situations.

\section{Assumptions And main Result}

We shall use the following standing assumption in order to guarantee the required properties of $F, \mathcal{D}(F)$ and $x^{\dagger}$.

Assumption 1 (Forward operator). The operator $F$ is weakly sequentially continuous, and its domain $\mathcal{D}(F)$ is convex and closed. Moreover, the solution $x^{\dagger} \in \mathcal{D}(F)$ is supposed to be an interior point of the domain $\mathcal{D}(F)$.

From (2) we have that the convex penalty functional $\|B(x-\bar{x})\|_{X}^{2}$ is stabilizing. Then, taking into account Assumption 1 we have that the Assumptions 3.11 and 3.22 in [26] are all fulfilled, and the usual assertions on existence and stability of regularized solutions (cf. [26, Section 4.1.1]) apply.

The unbounded self-adjoint operator $B$ satisfying (2) generates a Hilbert scale $\left\{X_{\tau}\right\}_{\tau \in \mathbb{R}}$ with $X_{0}=X, X_{\tau}=\mathcal{D}\left(B^{\tau}\right)$, and with corresponding norms $\|x\|_{\tau}:=\left\|B^{\tau} x\right\|_{X}$.

We make the following additional assumption on the structure of non-linearity for the forward operator $F$ with respect to the Hilbert scale generated by the operator $B$. Sufficient conditions and examples for this non-linearity assumption will be given in the appendix. 
Assumption 2 (Non-linearity structure). There is a number $a>0$, and there are constants $0<c_{a}<C_{a}<\infty$ such that

$$
c_{a}\left\|x-x^{\dagger}\right\|_{-a} \leq\left\|F(x)-F\left(x^{\dagger}\right)\right\|_{Y} \leq C_{a}\left\|x-x^{\dagger}\right\|_{-a} \text { for all } x \in \mathcal{D} .
$$

At this point we recall the concept of local well-posedness and illposedness from [13, Definition 2]. A similar concept is also mentioned in $[17]$.

Definition (local well-posedness and ill-posedness). The operator equation (1) with forward operator $F: \mathcal{D}(F) \subset X \rightarrow Y$ is called locally well-posed at the point $x^{\dagger} \in \mathcal{D}(F)$ if there is a ball $\mathcal{B}_{r}\left(x^{\dagger}\right)$ with center $x^{\dagger}$ and radius $r>0$ such that, for every sequence $\left\{x_{n}\right\}_{n \in \mathbb{N}} \subset$ $\mathcal{B}_{r}\left(x^{\dagger}\right) \cap \mathcal{D}(F)$, the implication

$$
\lim _{n \rightarrow \infty}\left\|F\left(x_{n}\right)-F\left(x^{\dagger}\right)\right\|_{Y}=0 \Longrightarrow \lim _{n \rightarrow \infty}\left\|x_{n}-x^{\dagger}\right\|_{X}=0
$$

is valid, otherwise (1) is called locally ill-posed at the point $x^{\dagger}$.

If $F=A \in \mathcal{L}(X, Y)$ is a bounded linear operator, then we note that (1) is locally well-posed everywhere if $F$ is injective and the range $\mathcal{R}(A)$ is a closed subset of $Y$, otherwise the equation is locally illposed everywhere. For linear $F=A$ the left inequality of (5) yields injectivity. For non-linear $F$ this left inequality of (5), which was also exploited in the paper [27], yields local injectivity at $x^{\dagger}$ and if the lower index $a$ were zero, then we would have local well-posedness of (1) from (5). But for $a>0$, i.e., when the norm in $X_{a}$ is weaker than the norm in $X$, local ill-posedness can occur and motivates the use of regularization for the stable approximate solution of (1).

We turn to the notion of solution smoothness, and we shall measure this with respect to the operator $B$.

Assumption 3 (Solution smoothness). There are $0<p<1$ and $E<\infty$ such that $x^{\dagger} \in \mathcal{D}\left(B^{p}\right)$ and

$$
x^{\dagger}-\bar{x} \in M_{p, E}:=\left\{x \in X_{p}, \quad\|x\|_{p}:=\left\|B^{p} x\right\|_{X} \leq E\right\}
$$

for the smooth reference element $\bar{x} \in \mathcal{D}$ from the Tikhonov functional $T_{\alpha}^{\delta}$. However, we assume that $x^{\dagger} \notin \mathcal{D}(B)$.

The focus in this study is to discuss oversmoothing with $x^{\dagger}-\bar{x} \in M_{p, E}$ for some $0<p<1$, but $x^{\dagger} \notin \mathcal{D}(B)$, albeit the Tikhonov penalty uses the operator $B$, such that all minimizers of (4) are smoother than the solution element $x^{\dagger}$.

Throughout this paper, we choose the regularization parameter a posteriori as $\alpha_{\mathrm{DP}}=\alpha_{\mathrm{DP}}\left(\delta, y^{\delta}\right)$ according to a specific version of the discrepancy principle, which is introduced as follows:

Discrepancy principle. For a prescribed constant $C>1$ chose the regularization $\alpha=\alpha_{D P}$ according to

$$
\left\|F\left(x_{\alpha}^{\delta}\right)-y^{\delta}\right\|_{Y}=C \delta .
$$


In the sequel we assume that $\alpha_{\mathrm{DP}}$ exists for the given data $y^{\delta} \in Y$, at least in the case of sufficiently small $\delta>0$. In our context (cf. (4)), the condition

$$
\left\|F(\bar{x})-y^{\delta}\right\|_{Y}>C \delta
$$

is sufficient for this existence in the special case of linear forward operators $F$. Due to possibly occurring gaps the existence of $\alpha_{\text {DP }}$ can fail for non-linear $F$, and additional conditions (cf. [2, Condition 3.9]) are required for ensuring the existence of $\alpha_{\mathrm{DP}}$.

In the subsequent analysis we suppose that the chosen parameter $\alpha=$ $\alpha\left(\delta, y^{\delta}\right)$ taken from the discrepancy principle as $\alpha=\alpha_{\mathrm{DP}}$ tends to zero as $\delta \rightarrow 0$. As the following lemma shows, this is no additional requirement, but it is ensured under the above assumptions.

Lemma 1. Under the assumptions stated above the regularization parameters $\alpha_{\mathrm{DP}}=\alpha_{\mathrm{DP}}\left(\delta, y^{\delta}\right)$, depending on $\delta$ and $y^{\delta}$, chosen according to the discrepancy principle, tend to zero when $\delta \rightarrow 0$ and $y^{\delta}$ obeys the noise model (3).

Proof. Consider a sequence $\delta_{n} \rightarrow 0$, and an associated sequence of noisy data $y^{\delta_{n}} \in Y$ with $\left\|F\left(x^{\dagger}\right)-y^{\delta_{n}}\right\|_{Y} \leq \delta_{n}$. Let $\alpha_{n}$ be the obtained sequence of regularization parameters according to the discrepancy principle, hence with $\left\|F\left(x_{\alpha_{n}}^{\delta_{n}}\right)-y^{\delta_{n}}\right\|_{Y}=C \delta_{n}$.

Suppose to the contrary that there is $\underline{\alpha}>0$ such that $\alpha_{n} \geq \underline{\alpha}$ for all $n \in \mathbb{N}$. Because $\bar{x} \in \mathcal{D}(F) \cap \mathcal{D}(B)$, all regularized solutions with regularization parameter $\underline{\alpha}>0$ and data $y^{\delta_{n}}$ satisfy the inequality

$$
\left\|F\left(x_{\underline{\alpha}}^{\delta_{n}}\right)-y^{\delta_{n}}\right\|_{Y}^{2}+\underline{\alpha}\left\|B\left(x_{\underline{\alpha}}^{\delta_{n}}-\bar{x}\right)\right\|_{X}^{2} \leq\left\|F(\bar{x})-y^{\delta_{n}}\right\|_{Y}^{2},
$$

which implies that there is some $K>0$ such that $m\left\|x_{\underline{\alpha}}^{\delta_{n}}-\bar{x}\right\|_{X} \leq$ $\left\|B\left(x_{\underline{\alpha}}^{\delta_{n}}-\bar{x}\right)\right\|_{X} \leq K$. Hence, there is a weakly convergent sub-sequence $x_{\underline{\alpha}}^{\delta_{n}} \longrightarrow z$ in the Hilbert space $X_{1}=\mathcal{D}(B)$. The limit element $z$ is also the weak limit in $\mathrm{X}$ and belongs to $\mathcal{D}(F) \cap \mathcal{D}(B)$, because the norm is lower semi-continuous and $\mathcal{D}(F)$ is weakly closed. Then the nondecreasing property of the discrepancy norm in Tikhonov regularization with respect to $\alpha$, the weak sequential continuity of $F$ and the local injectivity of $F$ at $x^{\dagger}$ expressed by the left inequality of (5) together with the properties $z \in \mathcal{D}(B)$ and $x^{\dagger} \notin \mathcal{D}(B)$ allow us to bound as follows:

$$
\begin{gathered}
0=\liminf _{k \rightarrow \infty} C \delta_{n_{k}}=\liminf _{k \rightarrow \infty}\left\|F\left(x_{\alpha_{n_{k}}}^{\delta_{n_{k}}}\right)-y^{\delta_{n_{k}}}\right\|_{Y} \\
\geq \liminf _{k \rightarrow \infty}\left\|F\left(x_{\underline{\alpha}}^{\delta_{n_{k}}}\right)-y^{\delta_{n_{k}}}\right\|_{Y}=\left\|F(z)-F\left(x^{\dagger}\right)\right\|_{Y}>0 .
\end{gathered}
$$

This is a contradiction, and completes the proof of the lemma.

Remark 1. It can be seen from the reasoning in the above proof, that under the present assumptions the penalty $\left\|B\left(x_{\alpha}^{\delta}-\bar{x}\right)\right\|_{X}^{2}$ must tend to infinity for the corresponding $\alpha=\alpha_{\mathrm{DP}}$, provided that $\delta \rightarrow 0$. 
The main result is the following.

Theorem. Consider the operator equation $F(x)=y$ in Hilbert spaces, with forward operator $F: \mathcal{D}(F) \subset X \rightarrow Y$ which obeys Assumptions 1 and 2. Let $x_{\alpha}^{\delta}$ be the minimizer of the Tikhonov minimization problem (4), where the regularization parameter $\alpha=\alpha_{\mathrm{DP}}$ is chosen according to the discrepancy principle. If $x^{\dagger} \in \mathcal{D}(F)$ has smoothness as in Assumption 3 for some $0<p<1$ (oversmoothing penalty), then $x_{\alpha}^{\delta}$ yields the convergence rate

$$
\left\|x_{\alpha}^{\delta}-x^{\dagger}\right\|=\mathcal{O}\left(\delta^{p /(a+p)}\right) \quad \text { as } \quad \delta \rightarrow 0 .
$$

We postpone a discussion of this result to Section 4, and we first prove the theorem in the next section.

\section{Proof of the MAIN RESUlT}

In various places we shall use interpolation inequalities in the Hilbert scale, which are generated by the operator $B$, see e.g. [8, Prop. 8.19]. These inequalities ensure, for given numbers $-a<r \leq s$, that we obtain

$$
\|x\|_{r} \leq\|x\|_{s}^{\frac{r+a}{s+a}}\|x\|_{-a}^{\frac{s-r}{s+a}}, \quad x \in X_{s}=\mathcal{D}\left(B^{s}\right) .
$$

In a first step we bound the error in the weak norm exploiting the lower bound of the inequality chain (5).

Lemma 2. Suppose that the Assumptions 1 and 2 hold. If the regularization parameter $\alpha=\alpha_{\mathrm{DP}}$ is chosen according to the discrepancy principle then

$$
\left\|x_{\alpha}^{\delta}-x^{\dagger}\right\|_{-a} \leq\left(\frac{C+1}{c_{a}}\right) \delta .
$$

We mention the following consequence.

Proposition 1. Let $\alpha=\alpha_{\mathrm{DP}}$ be chosen according to the discrepancy principle. Suppose that $\left\|B^{p}\left(x_{\alpha}^{\delta}-\bar{x}\right)\right\|_{X} \leq \tilde{E}$ holds true for some constant $\tilde{E}>0$. Then we have under Assumptions 1-3 that

$$
\left\|x_{\alpha}^{\delta}-x^{\dagger}\right\|_{X} \leq(E+\tilde{E})^{a /(a+p)}\left(\frac{C+1}{c_{a}} \delta\right)^{p /(a+p)} .
$$

Proof. From the bound on $\left\|B^{p}\left(x_{\alpha}^{\delta}-\bar{x}\right)\right\|_{X}$ and Assumption 3 we find that $\left\|x_{\alpha}^{\delta}-x^{\dagger}\right\|_{p} \leq E+\tilde{E}$. Now we use the interpolation inequality (8) with $r=0, s=p$, yielding

$$
\left\|x_{\alpha}^{\delta}-x^{\dagger}\right\|_{X} \leq\left\|x_{\alpha}^{\delta}-x^{\dagger}\right\|_{p}^{a /(a+p)}\left\|x_{\alpha}^{\delta}-x^{\dagger}\right\|_{-a}^{p /(a+p)} .
$$

With lemma 2 this completes the proof.

We thus make the important observation that it is enough to show that $\left\|B^{p}\left(x_{\alpha}^{\delta}-\bar{x}\right)\right\|_{X} \leq \tilde{E}$ holds true as $\delta \rightarrow 0$ and hence $\alpha=\alpha_{\mathrm{DP}} \rightarrow 0$. 
3.1. Auxiliary linear problem and auxiliary element. For the subsequent analysis the following linear Tikhonov regularization will be important. Consider the Tikhonov functional

$$
T_{-a, \alpha}(x):=\left\|x-x^{\dagger}\right\|_{-a}^{2}+\alpha\|B(x-\bar{x})\|_{X}^{2}, \quad x \in X .
$$

Proposition 2. Suppose that $x^{\dagger} \in M_{p, E}$ for some $0<p<1$. Given $\alpha>0$ let $x_{\alpha}$ be the minimizer of $T_{-a, \alpha}$. Then

$$
\begin{aligned}
\left\|x_{\alpha}-x^{\dagger}\right\|_{X} & \leq E \alpha^{p /(2(a+1))} \\
\left\|B^{-a}\left(x_{\alpha}-x^{\dagger}\right)\right\|_{X} & \leq E \alpha^{(a+p) /(2(a+1))}, \\
\left\|B\left(x_{\alpha}-\bar{x}\right)\right\|_{X} & \leq E \alpha^{(p-1) /(2(a+1))},
\end{aligned}
$$

and

$$
T_{-a, \alpha}\left(x_{\alpha}\right) \leq 2 E^{2} \alpha^{(a+p) /(a+1)} .
$$

Moreover, we have that

$$
\left\|x_{\alpha}-\bar{x}\right\|_{p} \leq E, \text { and }\left\|x_{\alpha}-x^{\dagger}\right\|_{p} \leq E .
$$

Proof. The minimizer $x_{\alpha}$ has the explicit form

$$
x_{\alpha}=\bar{x}+\left(B^{-2 a}+\alpha B^{2}\right)^{-1} B^{-2 a}\left(x^{\dagger}-\bar{x}\right) .
$$

Introducing the bounded self-adjoint operator $H:=B^{-2(a+1)}$ we can rewrite this as $x_{\alpha}=\bar{x}+(\alpha I+H)^{-1} H\left(x^{\dagger}-\bar{x}\right)$. Hence, with

$$
r_{\alpha}(t)=\alpha /(t+\alpha), \quad t>0 \text {, }
$$

we find

$$
\left\|x_{\alpha}-x^{\dagger}\right\|_{X}=\left\|r_{\alpha}(H)\left(x^{\dagger}-\bar{x}\right)\right\|_{X} .
$$

Now we use that $x^{\dagger} \in M_{p, E}$. First, this yields

$$
\left\|x_{\alpha}-x^{\dagger}\right\|_{X} \leq E\left\|r_{\alpha}(H) H^{p /(2(a+1))}\right\|_{X \rightarrow X} \leq E \alpha^{p /(2(a+1))},
$$

since $r_{\alpha}(t) t^{q} \leq \alpha^{q}$ whenever $0<q \leq 1, t>0$, due to the qualification of linear Tikhonov regularization. Similarly, because of $0<p<1<a+2$, we obtain the estimate

$$
\begin{aligned}
\left\|B^{-a}\left(x_{\alpha}-x^{\dagger}\right)\right\|_{X} & \leq E\left\|B^{-(a+p)} r_{\alpha}(H)\right\|_{X \rightarrow X}=E\left\|r_{\alpha}(H) H^{(a+p) /(2(a+1))}\right\|_{X \rightarrow X} \\
& \leq E \alpha^{(a+p) /(2(a+1))},
\end{aligned}
$$

by using the qualification of Tikhonov regularization, again.

In the same manner we bound $\left\|B\left(x_{\alpha}-\bar{x}\right)\right\|_{X}$ as

$$
\left\|B\left(x_{\alpha}-\bar{x}\right)\right\|_{X} \leq E \alpha^{(p-1) /(2(a+1))},
$$

and the simple calculation

$$
\alpha\left\|B\left(x_{\alpha}-\bar{x}\right)\right\|_{X}^{2} \leq E^{2} \alpha^{(1+(p-1) /(a+1))}=E^{2} \alpha^{(a+p) /(a+1)}
$$

completes the bound for the Tikhonov functional $T_{-a, \alpha}$. For the final assertion we start with bounding $\left\|x_{\alpha}-\bar{x}\right\|_{p}$ as

$$
\left\|x_{\alpha}-\bar{x}\right\|_{p}=\left\|B^{p}(\alpha I+H)^{-1} H\left(x^{\dagger}-\bar{x}\right)\right\|_{X} \leq\left\|B^{p}\left(x^{\dagger}-\bar{x}\right)\right\|_{X \rightarrow X} \leq E,
$$


and similarly

$$
\left\|x_{\alpha}-x^{\dagger}\right\|_{p}=\left\|B^{p} r_{\alpha}(H)\left(x^{\dagger}-\bar{x}\right)\right\|_{X} \leq\left\|B^{p}\left(x^{\dagger}-\bar{x}\right)\right\|_{X} \leq E .
$$

The proof is complete.

Remark 2. From the first bound $\left\|x_{\alpha}-x^{\dagger}\right\|_{X} \leq E \alpha^{p /(2(a+1))}$ we see that, for $\alpha>0$ small enough, the element $x_{\alpha}$ from the auxiliary linear problem will belong to the domain $\mathcal{D}(F)$, because $x^{\dagger}$ is an inner point of $\mathcal{D}(F)$.

Since the approximations $x_{\alpha}^{\delta}$ are smoother than the solution element $x^{\dagger}$, with respect to the operator $B$ generating the Hilbert scale, we shall employ some intermediate element of higher smoothness. The principal idea of using such auxiliary elements was already mentioned in [24] (see also [8, Theorem 10.7] and [15, 19] with more connection to our approach). To this end we shall employ in this context an element $x_{\alpha}=x_{\text {aux }}$ from the family $x_{\alpha}(\alpha>0)$ of regularized solutions minimizing the functional (10), with properties listed in Proposition 2, but for the parameter $\alpha=\alpha_{\text {aux }}(\delta)$ depending on the noise level $\delta$ according to a specific discrepancy principle given below in formula (16).

Definition (Auxiliary element). Consider the family $x_{\alpha}(\alpha>0)$ of regularized solutions minimizing the functional (10) and let $\delta>0$ be sufficiently small such that there is some $\alpha=\alpha_{\text {aux }}(\delta)$ satisfying the equation

$$
\left\|F\left(x_{\alpha}\right)-F\left(x^{\dagger}\right)\right\|_{Y}=(C-1) \delta,
$$

with constant $C>1$ as used in (7). Then we call the corresponding element $x_{\text {aux }}:=x_{\alpha_{\text {aux }}}(\delta)$ auxiliary element.

The auxiliary element $x_{\text {aux }} \in \mathcal{D}(F)$ exists for sufficiently small $\delta>0$, because $x^{\dagger}$ is an inner point of $\mathcal{D}(F)$ and we have for $\alpha \rightarrow 0$ that $\left\|x_{\alpha}-x^{\dagger}\right\|_{X} \rightarrow 0$ and thus, as a consequence of the right inequality in (5), $\left\|F\left(x_{\alpha}\right)-F\left(x^{\dagger}\right)\right\|_{Y} \rightarrow 0$. Moreover, the element $x_{\text {aux }}$ is uniquely determined and has the following optimality properties.

Proposition 3. The auxiliary element obeys

$$
\begin{aligned}
\left\|x_{\mathrm{aux}}(\delta)-x^{\dagger}\right\|_{X} & \leq E^{a /(a+p)}\left(\frac{C}{c_{a}} \delta\right)^{p /(a+p)}, \\
\left\|B\left(x_{\mathrm{aux}}(\delta)-\bar{x}\right)\right\|_{X} & \leq E\left(\frac{C_{a} E}{C-1} \delta\right)^{\frac{p-1}{a+p}},
\end{aligned}
$$

and

$$
\left\|x_{\text {aux }}(\delta)-x^{\dagger}\right\|_{-a} \leq \frac{C-1}{c_{a}} \delta .
$$


Proof. The first assertion is an easy application of interpolation, similar to Proposition 1, using the bound from Lemma 2 with $C$ instead of $C-1$, and the bound (15) from Proposition 2.

For the second assertion we exploit the fact that, at the parameter $\alpha_{\text {aux }}(\delta)$ and by favour of the estimate (12), we have

$$
(C-1) \delta=\left\|F\left(x_{\mathrm{aux}}\right)-F\left(x^{\dagger}\right)\right\|_{Y} \leq C_{a}\left\|x_{\mathrm{aux}}-x^{\dagger}\right\|_{-a} \leq C_{a} E \alpha_{\mathrm{aux}}^{\frac{a+p}{2(a+1)}} .
$$

This provides us with the lower bound

$$
\alpha_{\text {aux }}(\delta) \geq\left(\frac{C-1}{C_{a} E} \delta\right)^{\frac{2(a+1)}{a+p}} .
$$

Plugging this bound into the estimate (13) we find that

$$
\left\|B\left(x_{\text {aux }}(\delta)-\bar{x}\right)\right\|_{X} \leq E\left(\frac{C-1}{C_{a} E} \delta\right)^{\frac{p-1}{a+p}},
$$

where we used that $0<p<1$. For the last assertion we use Definition of $x_{\text {aux }}$ and the (non-linearity) Assumption 2 to derive

$$
c_{a}\left\|x_{\mathrm{aux}}-x^{\dagger}\right\|_{-a} \leq\left\|F\left(x_{\mathrm{aux}}\right)-F\left(x^{\dagger}\right)\right\|_{Y}=(C-1) \delta,
$$

from which the proof can easily be completed.

Remark 3. It will be seen from Lemma 3, below, that a similar upper bound can be shown for the growth of the penalty $\left\|B\left(x_{\alpha}^{\delta}-\bar{x}\right)\right\|_{X}$.

3.2. Bounding the term $\left\|B^{p}\left(x_{\alpha}^{\delta}-\bar{x}\right)\right\|_{X}$. Our goal is to apply Proposition 1 , and we thus aim at proving that $\left\|B^{p}\left(x_{\alpha}^{\delta}-\bar{x}\right)\right\|_{X} \leq \tilde{E}$. Since we already know that the auxiliary element $x_{\text {aux }}$ obeys $\left\|x_{\text {aux }}-\bar{x}\right\|_{p} \leq E$, it is sufficient to obtain $\left\|x_{\text {aux }}-x_{\alpha}^{\delta}\right\|_{p} \leq \hat{E}$ such that $\tilde{E}=E+\hat{E}$.

Lemma 3. Let $C>1$ be the constant used in (7) as well as in (16) and let parameters $\alpha=\alpha_{\mathrm{DP}}$ be chosen according to the discrepancy principle. Then there is a constant $\hat{E}$ depending on $a, p, C, c_{a}, C_{a}$ and $E$, but not on the noise level $\delta$ for which

$$
\left\|B^{p}\left(x_{\mathrm{aux}}-x_{\alpha}^{\delta}\right)\right\|_{X} \leq \hat{E} .
$$

Proof. The proof will be done in two steps.

Inserting $x_{\text {aux }}$ into the Tikhonov functional (4) we find, by using that both $\alpha$ were chosen according to the discrepancy principle and $x_{\text {aux }}$ was chosen as in (16), that

$$
\begin{aligned}
(C \delta)^{2}+\alpha\left\|B\left(x_{\alpha}^{\delta}-\bar{x}\right)\right\|_{X}^{2} & \leq\left\|F\left(x_{\alpha}^{\delta}\right)-y^{\delta}\right\|_{Y}^{2}+\alpha\left\|B\left(x_{\alpha}^{\delta}-\bar{x}\right)\right\|_{X}^{2} \\
& \leq\left\|F\left(x_{\text {aux }}\right)-y^{\delta}\right\|_{Y}^{2}+\alpha\left\|B\left(x_{\text {aux }}-\bar{x}\right)\right\|_{X}^{2} \\
& \leq((C-1) \delta+\delta)^{2}+\alpha\left\|B\left(x_{\text {aux }}-\bar{x}\right)\right\|_{X}^{2} \\
& =(C \delta)^{2}+\alpha\left\|B\left(x_{\text {aux }}-\bar{x}\right)\right\|_{X}^{2},
\end{aligned}
$$


such that $\left\|B\left(x_{\alpha}^{\delta}-\bar{x}\right)\right\|_{X} \leq\left\|B\left(x_{\text {aux }}-\bar{x}\right)\right\|_{X}$. Using the triangle inequality we find

$\left\|B\left(x_{\mathrm{aux}}-x_{\alpha}^{\delta}\right)\right\|_{X} \leq\left\|B\left(x_{\mathrm{aux}}-\bar{x}\right)\right\|_{X}+\left\|B\left(x_{\alpha}^{\delta}-\bar{x}\right)\right\|_{X} \leq 2\left\|B\left(x_{\mathrm{aux}}-\bar{x}\right)\right\|_{X}$.

Now we use the interpolation inequality (8). Since $-a<p<1$ we have the estimate

$$
\left\|x_{\mathrm{aux}}-x_{\alpha}^{\delta}\right\|_{p} \leq\left\|x_{\mathrm{aux}}-x_{\alpha}^{\delta}\right\|_{1}^{\frac{a+p}{a+1}}\left\|x_{\mathrm{aux}}-x_{\alpha}^{\delta}\right\|_{-a}^{\frac{1-p}{a+1}} .
$$

For the first factor in (22) we apply inequality (21) and the corresponding bound (18) from Proposition 3. From the bound (19) in Proposition 3 and Lemma 2 we find that

$$
\left\|x_{\mathrm{aux}}-x_{\alpha}^{\delta}\right\|_{-a} \leq\left\|x_{\mathrm{aux}}-x^{\dagger}\right\|_{-a}+\left\|x_{\alpha}^{\delta}-x^{\dagger}\right\|_{-a} \leq\left(\frac{2 C}{c_{a}}\right) \delta,
$$

which allows bounding the second factor in (22). Overall this yields

$$
\left\|x_{\mathrm{aux}}-x_{\alpha}^{\delta}\right\|_{p} \leq\left(2 E\left(\frac{C_{a} E}{C-1} \delta\right)^{\frac{p-1}{a+p}}\right)^{\frac{a+p}{a+1}}\left(\left(\frac{2 C}{c_{a}}\right) \delta\right)^{\frac{1-p}{a+1}}=: \hat{E}
$$

where the constant $\hat{E}$ depends on $a, p, C, c_{a}, C_{a}$ and $E$, but not on the noise level $\delta$. Since we have from the estimate (15) in Proposition 3 (which holds for all $\alpha>0$, and hence also for $\alpha_{\text {aux }}$ ) that $\left\|x_{\text {aux }}-\bar{x}\right\|_{p} \leq E$, we conclude that $\left\|B^{p}\left(x_{\alpha}^{\delta}-\bar{x}\right)\right\| \leq \hat{E}+E$.

This allows us to apply Proposition 1, and it yields the order optimality of the reconstruction $x_{\alpha}^{\delta}$ as stated in the theorem.

\section{Discussion}

First, we mention that in the limiting case $p=1$ the order optimal convergence rate as established in the theorem is well-known also for the discrepancy principle (cf. Theorem 3.1 in [27]). In this situation, where the penalty is not oversmoothing, for the proof of the rate result only the left inequality of (5) is required, and no auxiliary elements are needed. For other regularization methods, which do not use a penalty, the rates as established in the theorem are known. This is the case for Landweber iteration, see e.g. [23], and for Newton-type methods, see [14].

The theorem also applies for linear operators, and in this case it extends the original result from [21] to the a posteriori parameter choice by the discrepancy principle.

We add the following observation. The lower bound from (20) was derived for the parameter choice according to the discrepancy principle. We notice that this bound coincides up to different constants with the a 
priori parameter choice $\alpha(\delta)=\alpha_{\text {apriori }}(\delta)$ established in [21] and obeys the limit condition

$$
\frac{\delta^{2}}{\alpha(\delta)} \rightarrow \infty \quad \text { as } \quad \delta \rightarrow 0
$$

In the conventional case, i.e., when $x^{\dagger} \in \mathcal{D}(B)$, we always have (also for the discrepancy principe, cf. [1, Proposition 8]) that

$$
\frac{\delta^{2}}{\alpha(\delta)} \rightarrow 0 \quad \text { as } \quad \delta \rightarrow 0
$$

Together with the fact that $\alpha(\delta) \rightarrow 0$ this provides the basis of the convergence theory for regularized solutions when $x^{\dagger} \in \mathcal{D}(B)$ (cf. [26, Section 4.1.2]). Within regularization with oversmoothing penalty, i.e., when $x^{\dagger} \notin \mathcal{D}(B)$, this cannot be expected and even the limit condition (23) seems to be possible in general.

We also highlight that the optimal rate as described here shows a delay of the saturation phenomenon, known for Tikhonov regularization. To exhibit this let us stick to the linear case, and let us take $B:=$ $\left(A^{*} A\right)^{-1}$, hence we have that $x^{\dagger} \in \mathcal{D}(B)$ exactly if $x^{\dagger} \in \mathcal{R}\left(A^{*} A\right)$. The link condition from Assumption 2 then holds for $a=1 / 2$, and the maximal rate (for $p=1$, i.e., no oversmoothing), as established in the present study is $\delta^{1 /(1+1 / 2)}=\delta^{2 / 3}$, even under the discrepancy principle. Without the smoothness promoting penalty, i.e., when the penalty is $\|x-\bar{x}\|_{X}^{2}$, then, under the discrepancy principle, the best possible rate is $\delta^{1 / 2}$, which is attained for maximal smoothness $p=1 / 2$, i.e., when $x^{\dagger} \in \mathcal{R}\left(A^{*}\right)$.

\section{ACKNOWLEDGMENT}

The research was financially supported by Deutsche Forschungsgemeinschaft (DFG-grant HO 1454/10-1).

\section{APPENDIX}

We collect assertions and examples which highlight that the nonlinearity condition (5) of Assumption 2 is valid in specific cases. For a more detailed discussion of the interplay between the occurring norms $\left\|F(x)-F\left(x^{\dagger}\right)-F^{\prime}\left(x^{\dagger}\right)\left(x-x^{\dagger}\right)\right\|_{Y},\left\|F(x)-F\left(x^{\dagger}\right)\right\|_{Y}$ and also $\left\|F^{\prime}\left(x^{\dagger}\right)\left(x-x^{\dagger}\right)\right\|_{Y}$ in the case of ill-posed problems we refer to [13].

We start from the following result, which is easily verified, and we omit the proof.

Proposition 4. Suppose that the operator $F$ is continuously Fréchet differentiable in a ball $\mathcal{B}_{r}\left(x^{\dagger}\right) \subset \mathcal{D}(F)$ with radius $r>0$ and let denote the Fréchet derivatives by $F^{\prime}(x), x \in \mathcal{B}_{r}\left(x^{\dagger}\right)$. If there are constants $0<$ $g_{a} \leq G_{a}<\infty$ and $0<k_{a}<K_{a}<\infty$ such that

$$
g_{a}\left\|B^{-a} h\right\|_{X} \leq\left\|F^{\prime}\left(x^{\dagger}\right) h\right\|_{Y} \leq G_{a}\left\|B^{-a} h\right\|_{X} \quad \text { for all } h \in X,
$$


and

$$
k_{a}\left\|F^{\prime}\left(x^{\dagger}\right)\left(x-x^{\dagger}\right)\right\|_{Y} \leq\left\|F(x)-F\left(x^{\dagger}\right)\right\|_{Y} \leq K_{a}\left\|F^{\prime}\left(x^{\dagger}\right)\left(x-x^{\dagger}\right)\right\|_{Y},
$$

for all $x \in \mathcal{B}_{r}\left(x^{\dagger}\right)$, then Assumption 2 holds with constants $c_{a}=g_{a} k_{a}$ and $C_{a}=G_{a} K_{a}$.

Remark 4 . The inequality chain (24) restricts the class of possible operators $B$ for generating the Hilbert scale under consideration. The positive constant a can be interpreted as degree of ill-posedness for the linearized problem $F^{\prime}\left(x^{\dagger}\right) x=y$. We mention that the condition (24) has already been used in [22, Assumption 2.1 (iv)]). Since $B$ is assumed to be unbounded with lower bound $m>0$ of the spectrum $\sigma(B)$, the operator $B^{-a}$ is bounded and injective with $\left\|B^{-a}\right\|_{X \rightarrow X} \leq \frac{1}{m^{a}}$ and $0 \in \sigma\left(B^{-a}\right)$. Consequently, $F^{\prime}\left(x^{\dagger}\right)$ in (24) must be injective with non-closed range and $0 \in \sigma\left(\left(F^{\prime}\left(x^{\dagger}\right)^{*} F^{\prime}\left(x^{\dagger}\right)\right)^{1 / 2}\right)$.

We close this section with two corollaries of Proposition 4 and corresponding examples, where the non-linearity condition from (25) can be proven in specific situations.

Corollary 1. Suppose that the operator $F$ is continuously Fréchet differentiable in a ball $\mathcal{B}_{r}\left(x^{\dagger}\right) \subset \mathcal{D}(F)$ with radius $r>0$. If, for a constant $0 \leq \eta<1$, the non-linearity condition of tangential cone type (cf. [8, Section 11.1])

$$
\left\|F(x)-F\left(x^{\dagger}\right)-F^{\prime}\left(x^{\dagger}\right)\left(x-x^{\dagger}\right)\right\|_{Y} \leq \eta\left\|F(x)-F\left(x^{\dagger}\right)\right\|_{Y}
$$

is satisfied for all $x \in \mathcal{B}_{r}\left(x^{\dagger}\right)$ then (25) holds with $k_{a}=1 /(1+\eta)$ and $K_{a}=1 /(1-\eta)$. Hence (24) and (26) imply that Assumption 2 is valid.

Example 1 (Exponential growth model). The following example for an exponential growth model was originally introduced by Groetsch in [11, Section 3.1]. Here we aim at identifying the time dependent growth rate $x(t)(0 \leq t \leq T)$ from observations of the size $y(t)(0 \leq t \leq T)$ of a population with $y(0)=y_{0}>0$ such that the initial value problem

$$
y^{\prime}(t)=x(t) y(t) \quad(0 \leq t \leq T), \quad y(0)=y_{0},
$$

is satisfied. For $X=Y=L^{2}(0, T)$ the forward operator attains the form

$$
[F(x)](t)=y_{0} \exp \left(\int_{0}^{t} x(\tau) d \tau\right) \quad(0 \leq t \leq T) .
$$

We note that the corresponding operator equation $F(x)=y$ is locally ill-posed everywhere in $X$. The operator $F$ is Fréchet differentiable on the whole Hilbert space $L^{2}(0,1)$ and possesses the Fréchet derivative

$$
\left[F^{\prime}\left(x^{\dagger}\right) h\right](t)=\left[F\left(x^{\dagger}\right)\right](t) \int_{0}^{t} h(\tau) d \tau \quad(0 \leq t \leq T), \quad h \in L^{2}(0, T),
$$


continuously mapping in $L^{2}(0, T)$. One easily verifies that

$$
\left\|F(x)-F\left(x^{\dagger}\right)-F^{\prime}\left(x^{\dagger}\right)\left(x-x^{\dagger}\right)\right\|_{Y} \leq K\left\|F(x)-F\left(x^{\dagger}\right)\right\|_{Y}\left\|x-x^{\dagger}\right\|_{X}
$$

holds with some constant $K>0$ for all $x \in X$. Hence, for $\mathcal{D}(F)=$ $\overline{\mathcal{B}_{r}\left(x^{\dagger}\right)}$ (closed ball around $x^{\dagger}$ with sufficiently small radius $r>0$ ) the condition (26) is satisfied with $0<\eta<1$.

Looking at $(27)$ it is reasonable to consider the integration operator $[J h](t):=\int_{0}^{t} h(\tau) d \tau(0 \leq t \leq T)$ mapping in $L^{2}(0, T)$, and the related Hilbert scale $\left\{X_{\tau}\right\}_{\tau \in \mathbb{R}}$ generated by the operator $B=\left(J^{*} J\right)^{-1 / 2}$. With respect to this scale, and since $0<\underline{c} \leq\left[F\left(x^{\dagger}\right)\right](t) \leq \bar{c} \leq \infty(0 \leq t \leq T)$ for the multiplier function in $F^{\prime}\left(x^{\dagger}\right)$, we find that (5) holds with $a=1$.

The range $\mathcal{R}\left(\left(J^{*} J\right)^{-p / 2}\right)$ is well-known as the fractional Sobolev space $H^{p}(0, T)$ for $0<p<1 / 2$. It is a subspace of $H^{p}(0, T)$ for $1 / 2 \leq p \leq 1$ (cf. [9, Thm. 2.1]). The source condition $x^{\dagger} \in \mathcal{D}\left(B^{p}\right)$ for $0<p<1$ in combination with $x^{\dagger} \notin \mathcal{D}(B)$ implies that the function $x^{\dagger}$ belongs to the Sobolev space $H^{p}(0, T)$, but not to $H^{1}(0, T)$.

Corollary 2. Suppose that the operator $F$ is continuously Fréchet differentiable in a ball $\mathcal{B}_{r}\left(x^{\dagger}\right) \subset \mathcal{D}(F)$ with radius $r>0$. If there is a family $M(x): X \rightarrow X$ of bounded linear operators which obey $\|M(x)-I\|_{X \rightarrow X} \leq c<1$ such that

$$
F^{\prime}(x)=M(x) F^{\prime}\left(x^{\dagger}\right) \quad \text { for all } \quad x \in \mathcal{B}_{r}\left(x^{\dagger}\right),
$$

then the estimates from (25) hold with constants $k_{a}=1-c$ and $K_{a}=$ $1+c$. Thus (24) and (28) imply the validity of Assumption 2.

Proof. By mean value theorem in integral form we have for $x \in \mathcal{B}_{r}\left(x^{\dagger}\right)$

$$
\begin{aligned}
& \left\|F(x)-F\left(x^{\dagger}\right)-F^{\prime}\left(x^{\dagger}\right)\left(x-x^{\dagger}\right)\right\|_{Y}=\left\|\int_{0}^{1}\left[F^{\prime}\left(x^{\dagger}+t\left(x-x^{\dagger}\right)\right)-F^{\prime}\left(x^{\dagger}\right)\right]\left(x-x^{\dagger}\right) d t\right\|_{Y} \\
& \leq\left\|\int_{0}^{1}\left[M\left(x^{\dagger}+t\left(x-x^{\dagger}\right)\right)-I\right] F^{\prime}\left(x^{\dagger}\right)\left(x-x^{\dagger}\right) d t\right\|_{Y} \leq c\left\|F^{\prime}\left(x^{\dagger}\right)\left(x-x^{\dagger}\right)\right\|_{Y}
\end{aligned}
$$

and hence by triangle inequality

$(1-c)\left\|F^{\prime}\left(x^{\dagger}\right)\left(x-x^{\dagger}\right)\right\|_{Y} \leq\left\|F(x)-F\left(x^{\dagger}\right)\right\|_{Y} \leq(1+c)\left\|F^{\prime}\left(x^{\dagger}\right)\left(x-x^{\dagger}\right)\right\|_{Y}$.

The condition (28) and variants of requirements on $M(x)$ have been discussed in [12] (see also in [16]), and we recall Example 4.1 from [12]:

Example 2 (Non-linear Hammerstein integral operator). We consider the spaces $X:=H^{1}[0,1]$ and $Y:=L^{2}(0,1)$ and in this context the non-linear integral operator $F: X \rightarrow Y$, which is defined as

$$
[F(x)](t)=\int_{0}^{t} \phi(x(s)) d s, \quad 0 \leq t \leq 1 .
$$


Then, for a sufficiently smooth real function $\phi$ and for a sufficiently small radius $r>0$, the condition $(28)$ is satisfied with $\|M(x)-I\|_{X \rightarrow X} \leq c<1$ for arbitrary $x^{\dagger} \in X$ whenever the derivative $\phi^{\prime}$ of the kernel function $\phi$ is uniformly bounded below by a positive constants for all arguments under consideration.

\section{REFERENCES}

[1] S. W. Anzengruber, B. Hofmann, and P. Mathé. Regularization properties of the sequential discrepancy principle for Tikhonov regularization in Banach spaces. Appl. Anal., 93(7):1382-1400, 2014.

[2] S. W. Anzengruber and R. Ramlau. Morozov's discrepancy principle for Tikhonov-type functionals with nonlinear operators. Inverse Problems, 26(2):025001, 17, 2010.

[3] K. Bredies and H. K. Pikkarainen. Inverse problems in spaces of measures. ESAIM Control Optim. Calc. Var., 19(1):190-218, 2013.

[4] M. Burger, J. Flemming, and B. Hofmann. Convergence rates in $\ell^{1}$ regularization if the sparsity assumption fails. Inverse Problems, 29:025013, $16,2013$.

[5] M. Burger and S. Osher. A guide to the TV zoo. In Level Set and PDE Based Reconstruction Methods in Imaging, volume 2090 of Lecture Notes in Math., pages 1-70. Springer, Cham, 2013.

[6] C. Clason and K. Kunisch. A measure space approach to optimal source placement. Comput. Optim. Appl., 53(1):155-171, 2012.

[7] I. Daubechies, M. Defrise, and C. De Mol. An iterative thresholding algorithm for linear inverse problems with a sparsity constraint. Communications in Pure and Applied Mathematics, 57(11):1413-1457, 2004.

[8] H. W. Engl, M. Hanke, and A. Neubauer. Regularization of Inverse Problems, volume 375 of Mathematics and its Applications. Kluwer Academic Publishers Group, Dordrecht, 1996.

[9] R. Gorenflo, Y. Luchko, and M. Yamamoto. Time-fractional diffusion equation in the fractional Sobolev spaces. Fract. Calc. Appl. Anal., 18(3):799-820, 2015.

[10] M. Grasmair, M. Haltmeier, and O. Scherzer. Sparse regularization with $l^{q}$ penalty term. Inverse Problems, 24(5):055020, 13, 2008.

[11] C. W. Groetsch. Inverse Problems in the Mathematical Sciences. Vieweg Mathematics for Scientists and Engineers. Vieweg, Braunschweig, 1993.

[12] M. Hanke, A. Neubauer, and O. Scherzer. A convergence analysis of the Landweber iteration for nonlinear ill-posed problems. Numer. Math., 72(1):2137,1995 .

[13] B. Hofmann and O. Scherzer. Factors influencing the ill-posedness of nonlinear problems. Inverse Problems, 10(6):1277-1297, 1994.

[14] Q. Jin. Error estimates of some Newton-type methods for solving nonlinear inverse problems in Hilbert scales. Inverse Problems, 16(1):187-197, 2000.

[15] Q. Jin and H. Zong-yi. On an a posteriori parameter choice strategy for tikhonov regularization of nonlinear ill-posed problems. Numerische Mathematik, 83(07):139-159, 1999.

[16] B. Kaltenbacher. A note on logarithmic convergence rates for nonlinear Tikhonov regularization. J. Inverse Ill-Posed Probl., 16(1):79-88, 2008.

[17] D. Lorenz and H. Worliczek. Necessary conditions for variational regularization schemes. Inverse Problems, 29(7):075016, 19, 2013.

[18] D. A. Lorenz. Convergence rates and source conditions for Tikhonov regularization with sparsity constraints. J. Inverse Ill-Posed Probl., 16:463-478, 2008. 
[19] S. Lu, S. V. Pereverzev, and R. Ramlau. An analysis of Tikhonov regularization for nonlinear ill-posed problems under a general smoothness assumption. Inverse Problems, 23(1):217-230, 2007.

[20] P. Mathé and U. Tautenhahn. Enhancing linear regularization to treat large noise. J. Inverse Ill-Posed Probl., 19(6):859-879, 2011.

[21] F. Natterer. Error bounds for Tikhonov regularization in Hilbert scales. $A p$ plicable Anal., 18(1-2):29-37, 1984.

[22] A. Neubauer. Tikhonov regularization of nonlinear ill-posed problems in Hilbert scales. Appl. Anal., 46(1-2):59-72, 1992.

[23] A. Neubauer. On Landweber iteration for nonlinear ill-posed problems in Hilbert scales. Numer. Math., 85(2):309-328, 2000.

[24] R. Plato. Optimal algorithms for linear ill-posed problems yield regularization methods. Numer. Funct. Anal. Optim., 11(1-2):111-118, 1990.

[25] O. Scherzer, M. Grasmair, H. Grossauer, M. Haltmeier, and F. Lenzen. Variational Methods in Imaging, volume 167 of Applied Mathematical Sciences. Springer, New York, 2009.

[26] T. Schuster, B. Kaltenbacher, B. Hofmann, and K. S. Kazimierski. Regularization Methods in Banach Spaces, volume 10 of Radon Series on Computational and Applied Mathematics. Walter de Gruyter, Berlin/Boston, 2012.

[27] U. Tautenhahn. Error estimates for regularized solutions of nonlinear ill-posed problems. Inverse Problems, 10(2):485-500, 1994.

[28] A. N. Tikhonov, A. S. Leonov, and A. G. Yagola. Nonlinear Ill-Posed Problems. Vol. 1, 2, volume 14 of Applied Mathematics and Mathematical Computation. Chapman \& Hall, London, 1998.

[29] X. Q. Wan, Y. B. Wang, and M. Yamamoto. Detection of irregular points by regularization in numerical differentiation and application to edge detection. Inverse Problems, 22(3):1089-1103, 2006.

[30] Y. B. Wang, X. Z. Jia, and J. Cheng. A numerical differentiation method and its application to reconstruction of discontinuity. Inverse Problems, 18(6):1461$1476,2002$.

Department of Mathematics, Chemnitz University of Technology, 09107 Chemnitz, Germany

E-mail address: bernd.hofmann@mathematik.tu-chemnitz.de

Weierstrass Institute for Applied Analysis and Stochastics, MohrenSTrasse 39, 10117 Berlin, Germany

E-mail address: peter.mathe@wias-berlin.de 